\title{
Interactive comment on "Modeling the integrated framework of complex water resources system considering economic development, ecological protection, and food production: A practical tool for water management" by Yaogeng Tan et al.
}

Anonymous Referee \#2

Received and published: 24 December 2020

This paper provides a framework to study economic development, ecological protection, and food productivity (EEF) nexus by integrating optimization and system dynamics. The model presented in the paper predicts major performance measures of the nexus for the Upper reaches of Guijiang River Basin (UGRB) in China. Although I agree with the comments posted by the editor and the first reviewer, I believe the paper suffers from some more significant issues.

First, it is not clear what the contribution of the paper is. Is the paper trying to introduce a new method? Is it trying to solve a problem? Is it trying to introduce a new 
theory? The paper needs to have a clear goal and then focus on that. If the goal is to introduce a new method, it should provide clear information on how optimization and system dynamics can be integrated. The current manuscript does tell us much about the integration process, as explained elegantly by the first reviewer. If the goal is to solve a problem, the problem should be defined. The current presentation provides some predictions about some measures and explains their dynamics, but it is unclear what problem it is trying to solve. Is the problem unsustainability? Is it depletion of resources? What are the research questions? What is the hypothesis that the paper aims to test? And then, it must provide some robust solutions. The paper has some scattered solutions in the discussion section, but they need to be organized and become the focus of the paper if the goal is to solve a problem. Finally, if the goal is to advance a theory, the whole writing should support that goal. The current manuscript aims to achieve all of these goals, and that makes it ambiguous.

Second, the paper is hard to follow mainly because its English writing is poor. There are many instances of grammatical and typing errors, as well as bad writing. I have listed some of the examples at the end of this note.

Third, the paper does not get the system dynamics terminology right. The method is mistakenly called "system dynamic," which is wrong. Another instance is the use of "systematics" as a misnomer of "systems perspective" or "systems relationships." Yet another example is the use of "cause-and-effect feedback loop" (Fig. 6 caption) instead of "causal loop diagram." Also, some statements in the manuscript make me nervous and skeptical about the authors' command of system dynamics modeling. They claim: "if the water supply increases at the same rate as water demand caused by increased socio-economic index, this feedback will be the positive feedback regulation that results in the polarization because the ecological water will be occupied and environmental protection will not be guaranteed." Note the use of "if" and "this feedback will be" in this statement. A feedback loop cannot be conditional; it is either positive or negative. The ambiguity of a feedback loop indicates a major deficiency in the model. 
Fourth, the presented model is very confusing. It is not clear if the model is trying to "characterize" the real system or represent an ideal situation. If the goal is to characterize the real system, then a system dynamics model would be sufficient. Agents of a real system rarely optimize things. If the goal is to represent an ideal situation (in a neoclassical sense), then the optimization model would be sufficient. I believe this confusion arises because the goal of the modeling is not defined adequately. As the first reviewer explained, the relationships between the optimization and the SD model need to be clarified.

Fifth, the presented model has some questionable assumptions. For example, it assumes that "The goal of the economic agent is aiming at increasing revenue of secondary and tertiary industries, as well as maintaining human wellbeing. It can be reflected by the minimum household and industrial [water] shortage" (lines 190-2). The minimum household and industrial water shortage cannot reflect the dynamics of industrial revenues and human wellbeing. Depending on the time frame, a system can maximize its economic gain regardless of water shortage. Another example of questionable assumptions appears in line 242: "the production of livestock is also in proportion to its water usage." This is simply wrong unless a Leontief function is used to represent this linear relationship, which is not. Finally, equation $2 \mathrm{c}$ aims to minimize a constant, which does not make sense!

Sixth, there are assumptions that limit the generalizability of the model. For example, "In the last stage, the continuous increase of the overload index stimulates the policymakers to alleviate the growth rate of population and GDP" (lines 513-4). Local or state governments in countries that do not follow a central decision-making regime cannot control economic and population growth as a function of ecological health. Even in such regimes, a significant effect of ecological health on economic and population growth is still a strong assumption.

Other, less critical issues: 1 . The paper is unnecessarily long. There are many repetitions in the text (e.g. the sentence "food system is the indispensable component for

Printer-friendly version

Discussion paper
Interactive

comment 
human lives" has repeated many times). The first three paragraphs of the introduction section explain how important sustainability is. This could reduce to one or two sentences! Similarly, the first three paragraphs of the discussion section talk about the importance of sensitivity analysis. They can reduce to two sentences. 2 . It is claimed that Fig. 1 represents a framework of sustainable development. The diagram in Fig. 1 simply shows some causal relationships between modeled variables. How can it represent a sustainable development framework? 3. In line 294, it is claimed that GDP and population always increase, which is simply wrong. 4. "Rational" in Table 1 should be "Reasonable." 5. "Lookup function solved by optimal model" in Table 2 do not add any valuable information. 6 . Avoid the use of hyperlinks in the text.

Examples of poor English writing: 1. line 71: "combing" 2. line 204: "alternating" 3. line 208: "improve air pollutions" 4. line 246: "\&" 5. line 310: "where OI, PI, Cl is" 6. line 465: "finally" (appears twice in a paragraph) 7. lines 469-470: "the economic growth will increase sharply to ensure the local economic development." (circular logic) 8. line 477: "It is easy to understand because . . " (what is easy to understand?) 9. lines 504-5: "the feedback linkage will take effect as that the growing rate of water supply of household and industry (Fig.9d) will miss the rate of water demand" 10. line 511: "the rest water" 11 . lines 633-4: "decision-makers are usually hard to choose which one is the better than another one." 12. line 650: "contribute" (should be "attribute"?) 13. line 654: "The parameter $\theta$ and $\alpha$ equal 0.25" 14. line 689: "These individual three items are also prominent aspects or disciplines that contain numerous basic principles." (hard to understand) 15. line 709: "adequately" (should be "adequate") 16. line 712-3: "We need to take advantage of the positive effect as much as possible of a certain model that is, although, usually double-sided." (hard to understand)

Interactive comment on Hydrol. Earth Syst. Sci. Discuss., https://doi.org/10.5194/hess-2020$461,2020$.

Printer-friendly version

Discussion paper 